\title{
ILCEA
}

Revue de l'Institut des langues et cultures

d'Europe, Amérique, Afrique, Asie et Australie

14 | 2011

Traduction et Ergonomie

\section{L'ergonomie, nouveau paradigme pour la traductologie}

Ergonomics as a New Paradigm for Translation Studies

\section{Élisabeth Lavault-Olléon}

\section{(QpenEdition \\ Journals}

Édition électronique

URL : http://journals.openedition.org/ilcea/1078

DOI : 10.4000/ilcea.1078

ISSN : 2101-0609

Éditeur

UGA Éditions/Université Grenoble Alpes

Édition imprimée

ISBN : 978-2-84310-203-5

ISSN : 1639-6073

Référence électronique

Élisabeth Lavault-Olléon, "L'ergonomie, nouveau paradigme pour la traductologie », ILCEA [En ligne], 14 | 2011, mis en ligne le 30 juin 2011, consulté le 30 avril 2019. URL : http://journals.openedition.org/ ilcea/1078; DOI : 10.4000/ilcea.1078

Ce document a été généré automatiquement le 30 avril 2019

(C) ILCEA 


\title{
L'ergonomie, nouveau paradigme pour la traductologie
}

\author{
Ergonomics as a New Paradigm for Translation Studies
}

\author{
Élisabeth Lavault-Olléon
}

1 Dans un système universitaire soucieux de l'insertion professionnelle de ses diplômés, les responsables de formation suivent de près l'évolution des métiers afin d'ajuster les contenus d'enseignement au contexte professionnel et au marché de l'emploi ${ }^{1}$. Or, le métier de traducteur a été radicalement transformé par l'accès généralisé et mondialisé aux technologies de l'information et de la communication, qu'il s'agisse de contenus numérisés, d'outils logiciels, de glossaires et autres ressources en ligne ou de réseaux de traducteurs. Par exemple, pour le spécialiste multilingue de la communication qu'est le traducteur', la facilité d'accès à un volume considérable d'informations en ligne a radicalement changé le rapport au travail.

Dans les cours de traduction technique dispensés avant 1995, la recherche documentaire nécessitait une formation sur place à la bibliothèque des sciences, où se trouvaient des collections telles que Les techniques de l'ingénieur, avec lesquelles il fallait se familiariser pour trouver l'information recherchée parmi des dizaines de volumes sur support papier. Le trajet et la consultation des ressources à la bibliothèque prenaient un temps non négligeable, réduit aujourd'hui à quelques clics de souris sans qu'il soit nécessaire de quitter son poste de travail. Pour respecter les délais de livraison, l'activité de traduction en soi, c'est-à-dire le temps réellement passé sur le transfert du sens d'un énoncé d'une langue dans une autre, en était finalement diminuée d'autant. Est-ce à dire que la tâche de traduction y a gagné aujourd'hui et que, grâce aux facilités apportées par la technologie, le traducteur dispose de plus de temps pour traduire et peut rendre des travaux de meilleure qualité ? C'est justement parce que la réponse à cette question est complexe et met en jeu des éléments qui ne sont pas habituellement traités en traductologie qu'il a paru opportun d'inviter à ce débat une autre discipline, l'ergonomie, susceptible d'apporter un nouvel éclairage sur le travail du traducteur. 
3 Le secteur linguistique est devenu un secteur clé de l'économie. Une étude commanditée par la Commission européenne a estimé pour l'année 2008 la valeur économique de ce secteur à 8,4 milliards d'euros ${ }^{3}$ dans l'UE, et prévoit une valeur doublée en 2015. Aux côtés de la formation et de l'industrie des langues, la traduction y tient une place prépondérante. Paradoxalement, c'est au moment où les besoins en traduction n'ont jamais été aussi grands que la question de la survie du traducteur est ouvertement posée. En effet, toutes les prévisions optimistes de forte croissance du secteur ne nous renseignent pas sur ce que seront le rôle et les conditions de travail du traducteur dans cette traduction de masse, où la traduction automatique (TA) prendra probablement la part du lion. Un signe qui ne trompe pas: on parle de plus en plus de «traducteur humain ", et depuis peu de "traducteur biologique " 4 , comme si le terme "traducteur " employé seul désignait forcément... un logiciel.

Analyser l'évolution du secteur du point du vue du traducteur «humain » est donc une nécessité vitale : a-t-il une place dans cette évolution et comment son métier va-t-il se transformer? Les réponses à ces questions sont essentielles pour les formateurs, puisque les étudiants actuels seront en pleine maturité professionnelle dans dix ans ou plus. C'est pourquoi le présent article abordera en premier lieu l'évolution des formations ellesmêmes, puisque celles-ci ont déjà su intégrer un ensemble de paramètres professionnels, avant de présenter les pistes de recherche qui les aideront à poursuivre leur adaptation.

\section{L'évolution des formations}

5 S'il est indéniable qu'il existe certaines aptitudes pour traduire, notamment l'aisance à trouver la formule et le mot justes, il est évident aussi que la traduction professionnelle s'enseigne, comme l'attestent les programmes de master d'universités et écoles qui ont su rendre leurs enseignements véritablement professionnels et affichent un taux d'insertion élevé sur le marché du travail. Ces masters sont le résultat d'une évolution qui a vu s'épanouir, depuis les années 1950, dans l'ordre et par relation directe de cause à effet, les échanges internationaux, les besoins en traduction et les formations de traducteurs. La définition, la construction et l'évaluation des compétences à enseigner ont évolué parallèlement. À l'indispensable maîtrise des langues, étrangères et maternelle, s'est d'abord ajouté le besoin de «savoir traduire » doublé d'un «savoir se documenter » et d'un «savoir rédiger». Mais ces compétences fondamentales se trouvent aujourd'hui modifiées, d'abord par l'utilisation de nouvelles ressources documentaires, et surtout par les outils d'aide à la traduction et à la rédaction qui modifient radicalement l'activité de traduction elle-même. D'autres savoir-faire sont également devenus indispensables.

6 L'approche par compétences s'impose désormais dans l'ensemble des secteurs éducatifs. Issue des organismes de qualification professionnelle et des recherches en didactique, elle permet de fixer des objectifs, de structurer les enseignements et de donner de la visibilité aux formations, répondant ainsi à la demande des étudiants, des familles, des organismes de tutelle et des secteurs professionnels. La notion de compétence, définie dans de nombreux documents de référence ${ }^{5}$, permet de dépasser la connaissance pour associer savoir, savoir-faire et savoir-être. Elle relie la connaissance, qui est de l'ordre du déclaratif, à un «faire » qui est de l'ordre du procédural. L'accent est mis sur le savoirfaire, et plus précisément sur savoir "passer à l'acte", agir en contexte, dans une situation et avec des objectifs définis. 
7 Depuis 2008, les formations professionnelles en traduction ont été dynamisées en Europe par le projet EMT (European Master in Translation). La demande en traducteurs hautement qualifiés s'étant accrue du fait des besoins grandissants des institutions de l'Union européenne et d'autres employeurs, la création du label EMT permet de « contribuer à l'amélioration des formations en traduction dans l'Union européenne, tout en favorisant la coopération et les échanges entre les établissements d'enseignement supérieur proposant des cursus en traduction ${ }^{6}{ }^{\prime}$. Il s'agit d'améliorer la formation des traducteurs afin de veiller à ce que les compétences des diplômés puissent s'adapter aux exigences des employeurs.

Partant de ce constat, les responsables de la Direction générale de la traduction (DGT) à la Commission européenne ont établi, en s'appuyant sur les travaux d'experts européens, professeurs et formateurs en traduction (Schäffner et Adab, 2000 ; Gouadec, 2002 ; PACTE, 2003, etc.), un référentiel qui détaille les compétences nécessaires pour exercer le métier de traducteur au sein des institutions de l'UE et pour travailler dans le domaine, en évolution rapide, de la communication multilingue et multimédia. Les experts du projet EMT ont ensuite, en 2009, attribué le label d'excellence EMT à 34 formations ${ }^{7}$ européennes sur la base des enseignements mis en œuvre pour développer ce profil de compétences et d'autres paramètres tels que les infrastructures et équipements, le profil universitaire et professionnel des formateurs et les chiffres d'insertion professionnelle. Les six compétences déclinées par l'EMT ${ }^{8}$ sont les suivantes:

- compétence en matière de prestation de service de traduction (tant dans sa dimension interpersonnelle de gestion de clientèle et de prestation que dans sa dimension de production d'une traduction répondant au service demandé et aux normes en vigueur, en incluant la révision et l'assurance qualité) ;

- compétence linguistique (en langue A - dite maternelle - et en langues B et C) ;

- compétence interculturelle (tant sociolinguistique que textuelle);

- compétence en matière d'extraction de l'information (efficacité de la recherche documentaire et terminologique);

- compétence thématique (du domaine traité par les textes à traduire, incluant la compétence à développer des connaissances) ;

- compétence technologique ou instrumentale (maîtrise des outils informatiques, notamment de TAO et TA).

9 On notera l'importance accordée à la compétence strictement professionnelle de prestation de service en traduction, autrement dit la compétence propre au métier de traducteur. C'est l'enseignement de cette compétence-là (la première citée) qui différencie clairement une formation professionnelle de traducteurs d'une formation qui utilise la traduction comme outil pédagogique. Elle s'accompagne de deux autres compétences qui dépassent elles aussi les compétences linguistiques et interculturelles que l'on pourrait qualifier de «classiques»: la compétence instrumentale ou technologique, qui inclut les techniques d'extraction de l'information en plus de la maîtrise des outils d'aide à la traduction, ainsi que la compétence thématique ou extralinguistique, qui inclut un ou plusieurs domaines de spécialité. Les experts de l'EMT insistent sur l'interdépendance des compétences et sur les aptitudes transversales tant cognitives que stratégiques qu'elles impliquent.

Cette évolution professionnelle des formations émane de l'analyse des besoins du marché, soutenue par des recherches universitaires encore trop peu nombreuses dans le domaine 
appliqué. Mais elle est toujours en marche et le présent article a pour objet de donner une impulsion pour d'autres recherches dans ce domaine.

\section{La dichotomie recherche - métiers}

11 Le monde universitaire vit encore, pour la majorité de ses sections de lettres et de langues, dans la perpétuation de considérations littéraires et esthétiques souvent associées à une ignorance des réalités professionnelles, voire à un mépris pour tout ce qui apparaît comme technique ou appliqué. La dichotomie entre les recherches et thèses valorisées par les instances universitaires disciplinaires et les besoins mentionnés plus haut est évidente. Les colloques organisés en France affichent en majorité des problématiques ayant peu de liens avec la réalité des métiers de la traduction, même littéraire.

Cependant, quatre secteurs tendent à s'affirmer, en rapport plus ou moins étroit avec les formations professionnelles. Le domaine des langues de spécialité (Lerat, 1995; Gotti, 2003), et notamment dans les champs technique et juridique, a acquis progressivement ses lettres de noblesse dans la sphère académique ${ }^{9}$, mais il porte peu sur l'activité de traduction professionnelle car il alimente surtout le secteur de l'enseignement des langues aux spécialistes d'autres disciplines (LANSAD). Il s'appuie donc davantage sur la didactique et l'ingénierie pédagogique. Le domaine de la terminologie, très lié à la traduction dans les années 1980, tend paradoxalement à s'en éloigner depuis quelques années en se rapprochant de la linguistique (Cabré, 1998 ; L'Homme, 2004) et « s'oriente vers de nouvelles applications, en particulier celle de la gestion des connaissances » (Humbley, 2011), dont l'extraction des connaissances, le traitement automatique des langues (comme auxiliaire de la traduction automatique), l'ontologie et l'intelligence artificielle. Plus généralement, les industries de la langue génèrent un foisonnement de recherches, en lien certain avec l'opération de traduction en tant que transfert linguistique, mais sans lien avec ses réalités culturelles et humaines. Enfin, seulement quelques foyers universitaires en France ont proposé des colloques sur les métiers de la traduction, tous portés par des formateurs concernés par le label EMT ${ }^{10}$. Ces dernières recherches sont souvent difficiles à classer d'un point de vue académique: elles s'affichent comme multilingues, car elles traitent de problématiques transversales et ne rentrent donc pas, en France, dans les sections disciplinaires du Conseil national des universités (CNU). Sans ignorer ni sous-estimer les aspects linguistiques de la traduction, elles se concentrent sur le processus de traduction dans ses aspects cognitifs, sociologiques et organisationnels ou sur des segments de l'activité peu étudiés comme la révision ou la gestion de la qualité. En tant que recherches appliquées, elles ont du mal à trouver un ancrage théorique pertinent, même si elles sont explicitement identifiées comme "translation profession research" dans la dernière ontologie des recherches en traductologie (Vandepitte, 2008). Les institutions européennes ont, elles, bien compris que l'amélioration de la formation des traducteurs était liée à la connaissance de l'évolution des métiers: depuis 2010, le réseau Erasmus OPTIMALE (Optimiser la formation de traducteurs professionnels dans une Europe multilingue) qui regroupe 65 partenaires européens, s'est donné, entre autres objectifs, celui de valoriser l'émergence de nouvelles recherches axées sur les métiers de la traduction dans le champ des études traductologiques ${ }^{11}$. 
13 La théorie interprétative de la traduction (Seleskovitch et Lederer, 1984 ; Israël et Lederer, 2006) et les théories fonctionnalistes (Nord, 1997) avec notamment la théorie du skopos (Reiss et Vermeer, 1984 ; Vermeer, 1996), qui constituent depuis longtemps le cadre de référence des recherches sur la traduction professionnelle, restent fondamentales pour analyser les pratiques et nourrir les formations. Elles s'enrichissent d'autres approches axées sur le processus («process-oriented») et visant l'éthique, les bonnes pratiques, les normes, l'évaluation de la qualité, le processus d'exécution des traductions, entre autres (Williams et Chesterman, 2002 ; Gouadec, 2001, 2005), mais elles s'avèrent insuffisantes pour analyser les problématiques nouvelles induites par l'utilisation massive des nouvelles technologies en traduction. C'est pourquoi un autre cadre théorique serait bienvenu, un nouveau paradigme qui permettrait d'interpréter la réalité des métiers de la traduction aujourd'hui et dans les vingt ans à venir.

\section{Le paradigme de l'ergonomie}

La représentation archétypale du traducteur frappe par sa grande unité : les tableaux représentant saint Jérôme le montrent dans des conditions matérielles plus qu'inconfortables (la grotte de l'ermite) mais baignant dans une sorte d'atemporalité mystique, travaillant seul sur une œuvre unique et sacrée, dans une perspective de durée (sa traduction de l'ancien testament, la Vulgate, qui a été utilisée pendant près de 15 siècles, a très certainement battu le record de longévité d'une traduction). Cette représentation symbolique était déjà contredite par les pratiques dès le $\mathrm{IX}^{\mathrm{e}}$ siècle par exemple, dans ce qu'on a appelé l'École de Bagdad, où des équipes de spécialistes arabes traduisaient ensemble les grands écrits des médecins grecs (Salama-Carr, 1990), et dans d'autres "écoles de traduction » prospères au Moyen Âge. Cependant, c'est cette représentation du traducteur qui reste gravée dans les esprits : on l'imagine travaillant dans la solitude, dans sa tour d'ivoire, essentiellement sur des textes littéraires ou sacrés.

15 La réalité d'aujourd'hui, c'est la prédominance du non-littéraire et du non-sacré, le contraire de l'unité et de l'atemporalité : un travail inscrit dans un engrenage temporel tyrannique et dans un schéma de production de documents devenant obsolètes à court terme (moins d'un an avant la prochaine version d'un logiciel, d'un jeu ou d'un produit). Un travail que le traducteur ne peut désormais mener à bien qu'environné d'outils, connecté au reste du monde et relié à la communauté des traducteurs en temps réel. Dans la grande majorité des cas, l'objet du travail n'est pas une œuvre mais des documents de plus en plus morcelés, voire des fragments, nécessitant des tâches variées et multiples. Enfin, le travail devient non seulement multiforme, multilingue et multimédia, mais également collaboratif et évolutif (les textes à traduire évoluant parfois en même temps que la traduction).

$\mathrm{Au}$ tableau de saint Jérôme agenouillé dans sa grotte se substitue l'image de Charlie Chaplin dans Les Temps modernes, minuscule figure humaine prise dans l'engrenage inexorable de machines complexes et perpétuellement en mouvement. Pour appréhender cette réalité, il convient donc de se pencher sur le nouveau rapport du traducteur à la tâche de traduction, sur sa relation de dépendance aux outils, sur ses nouvelles relations avec ses collaborateurs et collègues et sur l'environnement de travail inédit que ces paramètres constituent, en bref sur la relation entre le traducteur et ses moyens, méthodes et milieux de travail. 

d'ergonomie (1969) : «L'étude scientifique de la relation entre l'homme et ses moyens, méthodes et milieux de travail». La dernière définition de l'IEA (International Ergonomics Association), datant de 2000, est la suivante :

L'ergonomie (ou Human Factors) est la discipline scientifique qui vise la compréhension fondamentale des interactions entre les humains et les autres composantes d'un système et la profession qui applique principes théoriques, données et méthodes en vue d'optimiser le bien-être des personnes et la performance globale des systèmes. (Lancry, 2009, p. 20)

Cette dualité d'une discipline associant une conception théorique et méthodologique et ses applications pratiques n'est pas sans rappeler la dichotomie souvent signalée entre les traductologues et les traducteurs, entre ceux qui analysent et ceux qui agissent, entre ceux qui parlent de traduction et ceux qui en font. Le principe de l'ergonomie, c'est justement d'ancrer la théorie dans l'observation du réel, afin de pouvoir proposer une remédiation. C'est bien ainsi que se posent certains traductologues, qui défendent une praxéologie (Ladmiral, 1979, p. 211) ou une traductologie fonctionnelle (Lavault-olléon, 2006, p. 244).

\section{Valeurs et critères de base en ergonomie}

19 En quoi cette discipline peut-elle nous aider à appréhender plus finement l'évolution des métiers de la traduction? Dépassant largement la conception restreinte qui la limite à l'étude de la hauteur des tables ou à la forme des boutons, l'ergonomie mise d'abord sur la prédominance du facteur humain et sur le besoin d'adapter le travail à l'homme et non le contraire. D'après Pierre Falzon (2004, p. 20), «l'ergonomie développe une approche holistique de l'homme, où celui-ci est simultanément pensé dans ses dimensions physiologiques, cognitives et sociales». Choisir cette position de base en traductologie signifie clairement que c'est le traducteur humain qui est au centre des recherches et que c'est cette activité humaine complexe qui est étudiée en situation, plutôt que l'outil (les logiciels de traduction automatique ou les mémoires de traduction) ou le processus et ses différents procédés (le transfert des éléments linguistiques et culturels du contenu à traduire).

20 Il est cependant évident que le facteur humain diffère selon qu'on se place du point de vue des organisations ou des personnes (Falzon, 2004, p. 23). Pour les premières, l'objectif est d'améliorer la performance (efficacité, productivité, fiabilité, durabilité, etc.) ; pour les secondes, il s'agit d'améliorer leur sécurité, leur santé, leur confort et leur satisfaction au travail, ainsi que la facilité d'usage des objets et des produits. Le double objectif paradoxal de l'ergonomie est la conjonction de deux logiques qui s'affrontent :

La logique technico-organisationnelle s'appuie sur une vision du travail comme vecteur de développement et de richesse qui tend à atteindre des objectifs économiques, financiers et aussi politiques. La logique du vivant est celle de l'agent, du travailleur, chargé par son activité de travail de participer à atteindre les objectifs prescrits par l'organisation du travail. (Hubault, 1992, cité par Landry, 2009, p. 7)

Cette problématique de base de l'ergonomie, allier efficacité et bien-être, rejoint le double objectif paradoxal du traducteur d'aujourd'hui : allier rentabilité et qualité, cette dernière ne pouvant être atteinte que si certaines conditions de travail, y compris de confort au 
travail, sont réunies. La relation de cause à effet entre les deux pôles est résumée par la formule du compromis ergonomique : « on est meilleur quand on est mieux ».

Dans les théories de l'action et de l'activité que mettent en avant les ergonomes, on note que « l'activité se construit [...] sur la base d'interactions locales, dans un contexte et des circonstances matérielles et particulières» (Darses, Falzon et Munduteguy, 2004, p. 197), elle est donc "située», conditionnée par un contexte et des interactions relativement ponctuelles. Cette prépondérance du contexte fait écho aux principes de la théorie du skopos, où l'activité du traducteur se définit prioritairement par rapport à la mission que doit remplir la traduction, telle que définie lors de l'échange avec le commanditaire.

La distinction entre tâche et activité, autre fondement de l'ergonomie, est également très pertinente pour la traduction. Elle permet de dissocier la tâche de traduction telle qu'elle est pensée, prescrite et attendue par le commanditaire et l'activité que déploie le traducteur pour l'effectuer, car « l'activité est la réponse que l'individu met en œuvre pour réaliser la tâche» (Rabardel et al., 2007, p. 27). L'activité est alors vue comme «le produit de l'interaction entre un sujet (l'agent), un objet (le motif) et des actions (processus sous-tendus par une activité mentale)»(Landry, 2009, p. 36). Dans celle-ci intervient un ensemble de paramètres fort éloignés de la représentation sommaire que peut avoir un profane (le commanditaire, par exemple), qui ne voit que le but à atteindre et néglige l'opérateur. L'activité du traducteur comporte un ensemble d'opérations cognitives complexes allant de l'extraction d'informations thématiques ou terminologiques à la révision, en passant par l'ensemble des opérations de transfert linguistique et culturel proprement dites. Elle s'analyse aussi à partir de comportements (gestes, postures, déplacements) et des contraintes et modes opératoires spécifiques à la situation de travail. Elle met en jeu la relation du traducteur aux outils, son comportement face à la technologie en général, les rapports entretenus avec l'équipe, qu'ils s'agissent de pairs (autres traducteurs) ou de supérieurs (chef de projet), ses états émotionnels, la relation au travail dans son ensemble. L'activité se manifeste aussi par un schéma organisationnel, un rapport au temps et aux normes. On devine aisément quel impact ces éléments peuvent avoir sur la qualité du produit livré et comment une meilleure compréhension de ces éléments pourrait améliorer cette dernière.

\section{Les méthodes et les domaines de l'ergonomie à appliquer à la traduction}

Certaines recherches traductologiques reprennent déjà en grande partie les valeurs et méthodes de ce paradigme que représente l'ergonomie. Le but du présent article est de montrer en quoi ces recherches existantes rentrent dans ce cadre, et aussi de proposer de creuser d'autres pistes allant dans ce sens, sans jamais perdre de vue l'intérêt de ces recherches pour la formation.

Les méthodes de l'ergonomie comprennent une double dimension: l'analyse d'une situation de travail en constitue la partie théorique ; la transformation, ou l'amélioration, de cette situation en est l'application professionnelle. L'analyse peut être conduite à différents niveaux : au niveau macroscopique du travail, des normes et des objectifs, ou au niveau de l'analyse plus fine de chaque situation particulière (mesures des conditions, des capacités cognitives, des effets sur les personnes, de la qualité, etc.). 

traduction pour agir sur elle. L'analyse peut être globale, centrée par exemple sur les nouvelles normes et leur impact sur l'activité : ainsi la norme CEN 15038 qui instaure l'obligation de relecture par un tiers pour les prestataires de services en traduction a eu un impact positif sur l'organisation du travail en poussant les traducteurs indépendants à nouer des relations plus étroites avec leurs pairs afin de faire relire leurs textes en échange du même service réciproque. La confrontation des objectifs du travail à la mesure de la qualité est un autre type de recherche mettant en évidence la relativité des niveaux de qualité et la perte de productivité que constitue la sur-qualité (Gouadec, 2001). Mais l'analyse peut aussi viser une granularité plus fine: par exemple, la mesure des rythmes de travail et de leur impact sur la production (ou sur le taux de renouvellement des personnels dans certaines sociétés de traduction), ou encore les recherches sur les processus cognitifs mis en œuvre en fonction de l'environnement de travail (Fougner Rydning, 2008).

C'est par l'observation des comportements au travail, par les enquêtes sur le terrain et par les entretiens avec les intéressés que l'analyse d'une situation de travail peut être lancée. La diffusion de ce type de recherches permet de mieux préparer les étudiants à leur métier et probablement d'aider aussi les professionnels à acquérir un regard distancié sur leur activité.

Nous aborderons dans cette optique les trois grands domaines de l'ergonomie: l'ergonomie physique (postures, poste de travail, santé et sécurité), l'ergonomie cognitive (processus mentaux au travail) et l'ergonomie organisationnelle (organisation des tâches, nouvelles formes de travail). Associée à l'ergonomie cognitive, l'ergonomie du produit, qui inclut l'interface homme-machine et l'ergonomie documentaire, est également un domaine clé pour la traduction.

\section{Ergonomie physique et traduction}

L'ergonomie physique s'intéresse aux «caractéristiques anatomiques, anthropométriques, physiologiques et biomécaniques » de la personne au travail (Falzon, 2004, p. 19). Les conséquences du travail sur la santé, et notamment les maladies et accidents du travail, ont été étudiées dès le Moyen Âge, et même avant, dans le souci d'améliorer le rendement du travail, mais aussi de réduire les coûts de l'invalidité. Si l'ergonomie physique évoque surtout des souffrances associées à des activités particulièrement éprouvantes (travail dans les mines, à la chaîne, dans l'industrie chimique, etc.), elle concerne aussi tout un ensemble de troubles de la santé provoqués par des activités plus sédentaires (troubles visuels des bijoutiers, par exemple). Les métiers de la traduction impliquent aujourd'hui une extrême sédentarité, avec un poste de travail totalement informatisé. Tout est dorénavant à portée de clic, du document au client, en passant par la documentation et les dictionnaires.

Les problèmes de santé les plus courants sont ceux qui se retrouvent dans tous les emplois de bureau : problèmes liés à la posture assise (troubles du dos et des jambes), aux mouvements courants d'apparence anodine, comme la saisie sur clavier, dont la répétition conduit à des accumulations surprenantes : les doigts qui frappent les touches du clavier pour saisir les 2500 mots quotidiens déplacent environ $550 \mathrm{~kg}$ par jour, sans parler des kilomètres parcourus (Pineau, 2011, ci-après). Les traducteurs contraints de 
porter des bandages pour soulager la tendinite du poignet sont de plus en plus nombreux. La question du travail sur écran et des troubles visuels est également posée.

31 Pourquoi ces questions ne sont-elles jamais abordées, ni même envisagées dans une formation de traducteurs, et pourquoi néglige-t-on à ce point l'aspect physique du travail du traducteur qui passe huit heures ou plus assis devant son écran, les doigts sur le clavier? L'apprentissage ergonomique de la dactylographie ainsi que l'information sur les postures à respecter et les matériels adaptés pourraient faire partie d'une formation qui s'affiche comme professionnelle. Les solutions alternatives, comme le recours accru aux outils de reconnaissance de la parole, qui remettent la traduction dictée au goût du jour, mériteraient d'être examinées en termes de performance et de confort, en attendant que se développent les souris à pied ou faciales...

Beaucoup plus pernicieuse encore, la question de l'environnement physique au travail mériterait une enquête fouillée et des préconisations. Celui-ci comprend aussi bien l'ambiance sonore que l'ambiance lumineuse et thermique. Dans certaines sociétés de traduction, le travail en open space est la règle, créant une ambiance sonore et visuelle génératrice de stress, à laquelle s'ajoute parfois l'impression d'être constamment sous surveillance. Enfin, dans certains métiers de la traduction orale, notamment ceux des interprètes et respeakers, l'ergonomie physique inclut l'entraînement et la protection de la voix.

33 «Améliorer l'efficacité du travail humain d'un côté, diminuer la peine de l'homme au travail et prévenir les risques sur sa santé, de l'autre » (Laville, 2004, p. 37), cette double préoccupation de l'ergonomie s'applique au métier de traducteur. Même si les problèmes énoncés ici concernent aussi de nombreux métiers autres que la traduction, il serait utile de préparer nos étudiants à prendre au sérieux ces questions qui ont des répercussions sur le bien-être physique et mental des personnes, sur la productivité et la qualité du travail avec, au bout du compte, la satisfaction des individus dans la société en général.

\section{Ergonomie cognitive et traduction}

L'ergonomie cognitive s'intéresse aux «processus mentaux, tels que la perception, la mémoire, le raisonnement et les réponses motrices, dans leurs effets sur les interactions entre les personnes et d'autres composantes d'un système » (Falzon, 2004, p. 19). Ce sont ses premières études sur les interprètes en situation de travail qui ont permis à Danica Seleskovitch d'élaborer la théorie interprétative de la traduction (TIT), à partir d'une observation in vivo des processus à l'œuvre, au moyen d'enregistrements en cabine d'interprètes et à partir de l'étude des carnets de notes des interprètes (Seleskovitch, 1975). Depuis, les recherches sur les processus cognitifs en traduction ont visé, entre autres, à démonter les mécanismes de la lecture et de la compréhension, de la mémorisation et de la remémorisation, de la génération d'énoncés grâce à la recherche analogique et à la visualisation (Kussmaul, 2005; Asadi et Séguinot 2005; Fougner Rydning 2008). Les recherches sont effectuées par l'observation de l'activité traduisante via des enregistrements d'écrans, d'opérations sur clavier ou de mouvements des yeux (oculométrie) mais aussi par l'analyse des données verbales concomitantes au moyen de protocoles de verbalisation (TAP). Elles peuvent aussi s'appuyer sur des entretiens a posteriori et analyser des données verbales rétrospectives qui peuvent être des commentaires des enregistrements précédents (Massey et Ehrensberger-Dow, 2011, ciaprès). Ces méthodes existent depuis une dizaine d'années, mais ce n'est qu'assez 
récemment qu'elles portent sur la relation du traducteur à la technologie, prenant ainsi une orientation plus marquée vers l'ergonomie.

L'activité de traduction est le plus souvent étudiée par la comparaison de sujets aux niveaux de compétences différents et opérant dans des environnements de travail variés. L'observation de traducteurs expérimentés donne la possibilité d'analyser comment les processus cognitifs traductionnels sont modifiés par la multiplicité des ressources (et des fenêtres) à l'écran, par la segmentation des textes via les mémoires de traduction, par la complexité d'opérations qui combinent dans un même énoncé la révision de segments déjà traduits complètement ou en partie et la traduction de segments non présents dans la mémoire. Certaines recherches commencent à montrer comment les outils de TAO finissent par modeler les traducteurs et influencer leur façon de traduire (Mesa-Lao et Sanchez Gijón, 2011) ${ }^{12}$ ou à mettre en évidence les différences de comportements face à différents types d'outils (Carl, 2011).

Elles peuvent aussi avoir une visée didactique, en comparant des traducteurs débutants et expérimentés, ou en se concentrant sur les erreurs, les hésitations et les pertes de temps des traducteurs inexpérimentés, notamment par l'oculométrie (Massey et EhrensbergerDow, 2011, ci-après). Ces recherches sur les processus cognitifs démontrent que la qualité d'une traduction (et sa non-qualité) peut résulter d'éléments jusque-là occultés, comme les compétences (ou incompétences) instrumentales des traducteurs, à savoir leur aisance et efficacité dans la manipulation des outils, ce qui donne alors des indications précieuses aux formateurs. Cette compétence instrumentale dépend de la formation reçue, mais elle s'avère elle-même intimement liée à l'ergonomie des produits, de leur interface et de leurs fonctionnalités.

Pour que la démarche ergonomique soit complète, ces analyses devraient montrer quelles stratégies de remédiation sont mobilisées par les sujets pour s'adapter à leur nouvel environnement (que ce soit la traduction de segments sans contexte ou l'utilisation d'outils peu conviviaux, par exemple). Elles peuvent aussi mettre en évidence le besoin d'adapter les outils à leurs utilisateurs, et donc la nécessité pour les concepteurs de logiciels de consulter les traducteurs, ce qui n'est malheureusement pas toujours le cas. Enfin, au niveau de la production de documents elle-même, l'ergonomie documentaire est un élément indispensable de la traduction spécialisée, déjà bien analysé par les rédacteurs techniques qui en font le cœur de leur métier.

Les outils créent de nouvelles tâches: le perfectionnement constant de la TA depuis l'avènement de la traduction statistique et des méga-mémoires exige que l'on place l'activité de postédition au cœur de la formation, d'autant plus qu'il s'agit d'une activité complexe qui associe souvent dans un même document la révision de fragments produits par TA, par TAO à partir de mémoires, et par traduction humaine (Brunette et O'Brien, 2011, ci-après). Le besoin d'introduire la postédition dans les formations de traducteurs est avéré (O'Brien, 2002), mais l'organisation économique de la postédition est encore floue, sa reconnaissance en tant qu'activité spécifique n'est pas affirmée et son coût (à facturer) est inégalement maîtrisé. Une meilleure connaissance des compétences et stratégies mobilisées en postédition, de son organisation matérielle, des délais qu'elle nécessite et des degrés de qualité attendus permettrait aux traducteurs de l'accepter comme une tâche faisant partie intégrante de leur métier et aux formateurs de l'enseigner de façon plus organisée. 


\section{Ergonomie organisationnelle et traduction} nationales, qui résistent plus ou moins à l'automatisation étendue. Un bon exemple est la Direction générale de la traduction (DGT) à Bruxelles, avec ses 1750 traducteurs pour les 23 langues de l'Union européenne, qui bénéficient de ressources et d'un environnement de travail exceptionnels : il s'agit vraiment de traduction humaine assistée par la machine (THAO), avec le traducteur au centre, inséré dans une équipe de même langue maternelle travaillant à partir de différentes langues dans des conditions optimales (recrutement rigoureux, ressources humaines importantes, outils adaptés, délais raisonnables, révision valorisée, accueil et formation de stagiaires, etc.). Dans ces situations, l'ergonomie organisationnelle peut analyser la gestion des relations entre les personnes qui se divisent les tâches, par exemple la relation réviseur-révisé. Elle peut aussi porter sur les résistances à la modernisation et sur la façon dont l'introduction d'un système de TA peut être perçue, tolérée, puis éventuellement adoptée. grands prestataires de services linguistiques qui gèrent l'ensemble de la documentation, de la communication et de la traduction des grandes entreprises multinationales. Cellesci ont de tels besoins (par exemple la mise à jour quasi-permanente de sites web et de brochures sur leurs produits en 20 voire 35 langues) que seule l'automatisation permet de les satisfaire. Dans ce cas, un prestataire unique propose de réorganiser l'ensemble de la chaîne documentaire en centralisant la gestion de contenu (mémoires, archives) et en automatisant la majeure partie des processus (extraction et gestion automatique de la terminologie, mémoires de traduction, traduction automatique, assurance qualité). Le traducteur est soit une petite main perdue dans les rouages de l'automatisation, soit le valideur en bout de chaîne, qui s'assure de la qualité du produit livré au client, en prenant en compte l'adaptation culturelle nécessaire à la localisation. Un nouveau métier, mal connu dans les formations, qui met en œuvre de nouvelles compétences et nécessiterait une observation sur le terrain.

44

ILCEA, 14 | 2011 
petites sociétés de traduction, avec des traducteurs travaillant sur place, qui communiquent en temps réel et utilisent les outils de façon collaborative sur une plateforme en ligne permettant de travailler simultanément sur les mémoires multilingues d'un même document (Lavault-Olléon, 2008). L'association de la traduction humaine et de la technologie est alors positive tant pour les traducteurs que pour les commanditaires.

Mais la vraie révolution vient des outils collaboratifs ouverts, qui associent l'informatique en nuage (dite aussi dématérialisée) et l'externalisation ouverte (" crowd sourcing »). Ces dernières technologies gomment les repères géographiques ou socioprofessionnels : toute personne, où qu'elle soit et quelles que soient ses compétences, peut apporter sa pierre à l'édifice. C'est déjà le cas pour le "fansubbing » où les amateurs de séries audiovisuelles peuvent anticiper sur la traduction des sous-titres, ou le "ROMhacking ", où les amateurs de jeux vidéo sont non seulement incités à traduire gratuitement, par jeu ou par le biais de compétition ("translation hacking»), mais peuvent également modifier les jeux. Des sites aussi puissants (et riches) que Linkedin, Facebook et Twitter ont recours à leurs utilisateurs pour traduire gratuitement leur interface, de même que la totalité des logiciels open source qui sont par nature traduits par la "communauté». Si le travail gratuit peut sembler acceptable dans un environnement ludique ou gratifiant (par altruisme ou goût du piratage, par exemple), espère-t-on aussi se passer de traducteurs professionnels dans des domaines autres que l'industrie des loisirs ou le monde open source? La démarche touche également la postédition, comme en témoigne la traduction d'une encyclopédie réalisée par TA puis postéditée collaborativement et gratuitement par des experts du domaine (parfois étudiants), cette expérience ayant toutefois été organisée dans le cadre d'un projet de recherche, avec des postéditeurs sollicités, n'agissant donc pas de façon spontanée. Il semble difficile d'imaginer que le bénévolat puisse devenir la règle pour répondre à ce type de besoin. Le périmètre de ces activités bénévoles serait donc à mesurer rapidement : quel corpus sera le plus sujet à cette innovation, et quels textes ne s'y prêteront pas? On peut penser que la majorité des secteurs spécialisés, la traduction juridique notamment, resteront l'apanage des professionnels, la motivation ou l'expertise faisant alors défaut aux amateurs, et les experts du domaine n'ayant pas de temps à y consacrer.

Plus encore que l'automatisation, l'innovation technologique qu'apporte le « crowdsourcing" est donc à la base de modifications radicales de la profession de traducteur, qui posent aux chercheurs deux questions de fond: comment démontrer l'importance de la qualité d'une traduction d'une part, et comment souligner le lien indissociable entre professionnalisme et qualité d'autre part? De même que la généralisation sur la Toile de documents non conformes aux normes orthographiques et typographiques tend à augmenter la tolérance aux fautes d'orthographe, la généralisation de traductions faites par des amateurs peut à terme faire baisser l'exigence de qualité que défend la profession. Les recherches sur la perception et l'évaluation de la qualité des traductions, ainsi que sur les représentations que peuvent en avoir le grand public et les spécialistes, sont devenues indispensables pour valoriser les compétences professionnelles. C'est la question même de la professionnalisation d'une activité qui est posée. C'est aussi celle de l'évolution d'un métier qui devra inexorablement s'élargir à d'autres fonctions plus valorisées, comme l'assurance qualité et la médiation interculturelle, voire l'ingénierie multilingue. 


\section{Conclusion}

L'approche ergonomique permet de mettre au jour des aspects souvent oubliés par les chercheurs en traductologie, qui constituent un champ de recherche très riche dont l'exploitation est déjà bien amorcée. Ces recherches ont une visée pragmatique, puisqu'elles sont axées sur la formation. Elles pourraient aussi avoir pour ambition d'agir sur l'évolution des outils et des métiers, en dénonçant les effets indésirables de l'automatisation, tant sur la qualité des produits que sur le bien-être des personnes, et en proposant des modalités permettant de définir et de valoriser le facteur humain dans le processus de traduction et de gestion de contenus multilingues. La plus-value que représente l'intervention humaine dans la qualité du produit fini reste la meilleure justification de cet ingénieur en communication multilingue et multimédia qu'est devenu le traducteur (Gouadec, 2002). Pour la défendre, nous plaiderons pour que se développe une traductologie fonctionnelle qui tienne enfin compte du rôle essentiel que joue l'ergonomie sous toutes ses formes dans la qualité d'une traduction.

\section{BIBLIOGRAPHIE}

ASADI Paula et SÉGUINOT Candace, « Shortcuts, Strategies and General Patterns in a Process Study of Nine Professionals » [en ligne], Meta, vol. 50, n² 2, 2005, Les Presses de l'Université de Montréal, p. 522-547. Disponible sur <www.erudit.org/revue/META/2005/v50/n2/010998ar.html> [consulté le $1^{\text {er }}$ juin 2011].

BRUNETTE Louise et O'BRIEN Sharon, « Quelle ergonomie pour la pratique postéditrice des textes traduits?» [en ligne], ILCEA, $\mathrm{n}^{\circ} 14,2011$. Disponible dans le présent numéro.

CABRÉ Maria Teresa, La terminologie, Théorie, méthode et applications, Les Presses de l'Université d'Ottawa / Paris, Armand Colin, 1998, 322 p.

CARl Michael, Dragsted Barbara et JAKobSen Arnt Lykke, « On the Systematicity of Human Translation Processes », communication au colloque Tralogy (Paris, CNRS, 3-4 mars 2011), 2011 (actes à paraître).

DARSES Françoise, FAlzon Pierre et MunduteguY Christophe, « Paradigmes et modèles pour l'analyse cognitive des activités finalisées », dans P. FALzon (éd.), Ergonomie, Paris, Presses universitaires de France, 2004, p. 191-212.

Falzon Pierre (éd.), Ergonomie, Paris, Presses universitaires de France, 2004, 680 p.

FOUGNER RYDNING Antin, « La démarche de l'expert-traducteur face à un problème de reformulation » [en ligne], Meta, vol. 53, $\mathrm{n}^{\circ}$ 4, 2008, p. 748-764. Disponible sur <www.erudit.org/

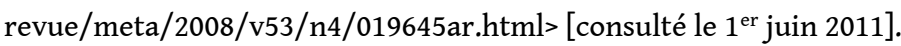

GoTTI Maurizio, Specialized Discourse. Linguistic Features and Changing Conventions, Berne, Peter Lang, 2003, $351 \mathrm{p}$. 
GOUADEC Daniel, « La qualité en traduction : types et modes de traduction », Actes de la septième université d'été en traduction, Paris, La Maison du dictionnaire, 2001, p. 9-70.

_, Profession : traducteur, Paris, La Maison du dictionnaire, 2002, 432 p.

-, « Modélisation du processus d'exécution des traductions », Meta, vol. 50, n 2, 2005, p. 643-655.

HuMBLEY John, « Terminologie et traduction, une complémentarité oubliée ? », communication au colloque Tralogy (Paris, CNRS, 3 mars 2011), 2011 (actes à paraître).

ISRAËL Fortunato et LEDERER Marianne, Théorie interprétative de la traduction, 3 tomes, Paris, Lettres Modernes, 2006.

Kussmaul Paul, «Translation through Visualization » [en ligne], Meta, vol. 50, $\mathrm{n}^{\circ}$ 2, 2005, p. 378-391. Disponible sur <www.erudit.org/revue/META/2005/v50/n2/010943ar.html> [consulté le $1^{\mathrm{er}}$ juin 2011].

L'Homme Marie-Claude, La terminologie, principes et techniques, Montréal, Les Presses de l'Université de Montréal, 2004, 280 p.

LADMIRAL Jean-René, Traduire : théorèmes pour la traduction, Paris, Gallimard, 1994 (1 ${ }^{\text {re }}$ édition 1979).

LANCRY Alain, L'Ergonomie, Paris, Presses universitaires de France, 2009, coll. « Que sais-je ?, $\mathrm{n}^{\circ}$ $1626 », 127 \mathrm{p}$.

LAVAULT-OlLÉon Élisabeth, «Traductologie et/ou professionnalisation », dans M. Ballard (éd.), Qu'est-ce que la traductologie ?, Artois Presses Université, 2006, p. 237-250.

-, « La TAO au secours du multilinguisme », Actes du Congrès mondial sur la traduction spécialisée, Cuba, 2008. Disponible sur <http://dtil.unilat.org/cmte2008/actas/Actas\%20CMTE.pdf> [consulté le $1^{\mathrm{er}}$ juin 2011].

LAVILLE Antoine, « Repères pour une histoire de l'ergonomie francophone », dans P. Falzon, Ergonomie, Paris, Presses universitaires de France, 2004, p. 37-50.

LERAT Pierre, Les langues spécialisées, Paris, Presses universitaires de France, 1995, 201 p.

MASSEY Gary et EHRENBERGER-Dow Maureen, « Technical and Instrumental Competence in Translation: Ergonomic Aspects of Workplace Processes and Practices » [en ligne], ILCEA, $\mathrm{n}^{\circ} 14$, 2011. Disponible dans le présent numéro.

MESA-LAO B. et SANCHEZ GIJÓN Pilar, « The way CAT tools mold translations - Final results of the TRACE project », communication au colloque Tralogy (Paris, CNRS, 3 mars 2011), 2011 (actes à paraître)

NORD Christiane, Translating as a Purposeful Activity - Functionalist Approaches Explained, Manchester, St Jerome Publishing, 1997, 154 p.

O'BRIEN Sharon, Teaching Post-editing: A Proposal for Course Content, 2002. Disponible sur <www.mtarchive.info/EAMT-2002-OBrien.pdf> [consulté le $1^{\mathrm{er}}$ juin 2011].

PACTE, « Building a translation competence model », dans I. Alves (dir.), Triangulating Translation, Amsterdam et Philadelphie, Benjamins, 2003, p. 43-66.

PINEAU Martine, « La main et le clavier : histoire d'un malentendu » [en ligne], ILCEA, nº 14, 2011. Disponible dans le présent numéro.

Rabardel Pierre, Carlin Nicole, Chesnais Marion, Lang Nathalie et PAscal Martine, Ergonomie : concepts et méthodes, Toulouse, Octarès Éditions, 2007 ( $1^{\text {re }}$ édition 1998), 180 p. 
REISS Katharina et VERMEER Hans, Grundlegung einer allgemeinen Translationstheorie, Tübingen, Niemeyer, 1984, 245 p.

SALAMA-CARR Myriam, La traduction à l'époque abbasside, Paris, Didier Érudition, 1990, coll.

« Traductologie, $\mathrm{n}^{\circ} 6$ ", $122 \mathrm{p}$.

SCHÄFFNER Christina et ADAB Beverley (éds), Developing Translation Competence, Amsterdam et Philadelphie, John Benjamins, 2000, coll. « Benjamins Translation Library, n 38 », 244 p.

SELESKOVITCH Danica, Langage, langues et mémoire, Paris, Minard, 1975, 272 p.

SELESKOVITCH Danica et LEDERER Marianne, Interpréter pour traduire, Paris, Didier Érudition, coll.

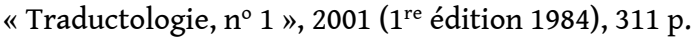

VANDEPITTE Sonia, «Remapping Translation Studies: Towards a Translation Studies Ontology » [en ligne], Meta, vol. 53, $n^{\circ}$ 3, 2008, p. 569-588. Disponible sur <www.erudit.org/revue/meta/2008/ v53/n3/019240ar.html> [consulté le $1^{\text {er }}$ juin 2011].

VERMEER Hans J., A Skopos Theory of Translation, Heidelberg, Textcontext Wissenschaft, 1996, 136 p. Williams Jenny et Chesterman Andrew, The Map. A Beginner's Guide to Doing Research in Translation Studies, Manchester, St Jerome Publishing, 2002, 149 p.

\section{NOTES}

1. L'auteur aborde la problématique de l'ergonomie en traduction en tant que directrice du master Traduction spécialisée multilingue de l'université Stendhal-Grenoble 3 depuis près de 20 ans, et soucieuse de l'insertion professionnelle des jeunes diplômés.

2. Dans le présent article, conformément aux normes du français, le masculin singulier «traducteur» représente le métier, même si celui-ci est majoritairement exercé par des traductrices.

3. Pour en savoir plus sur ce chiffre: "This figure comprises the industry sectors of translation, interpreting software localisation and website globalization, language technology tool development, language teaching, consultancy in linguistics issues and organization of international conferences with multilingual requirement " (Study on the size of the language industry in Europe, Language Technology Center (LTC) pour la Direction générale de la traduction (DGT), Commission 2009, p. III).

4. Ces termes ont été utilisés de façon récurrente au colloque Tralogy (Paris, CNRS, 3-4 mars 2011), où se trouvaient regroupés des traducteurs, des traductologues et des chercheurs en traitement automatique des langues (TAL) et en TA.

5. L'AFNOR définit la compétence comme une «combinaison de connaissances, savoir-faire, expériences et comportements s'exerçant dans un contexte précis, [qui] se constate lors de sa mise en œuvre en situation professionnelle, sociale ou de formation » (AFNOR X50-750).

6. Descriptif du Master européen en traduction (EMT) sur le site de la Direction générale de la traduction, Bruxelles. Disponible à l'adresse <http://ec.europa.eu/dgs/translation/programmes/ emt/index_fr.htm> [consulté le $1^{\text {er }}$ juin 2011].

7. En mai 2011, 20 nouvelles formations ont reçu le label EMT, portant le total à 54 .

8. Compétences pour les traducteurs professionnels, experts en communication multilingue et multimédia, établies par le groupe d'experts EMT, janvier 2009. Document disponible et téléchargeable à l'adresse <http://ec.europa.eu/dgs/translation/programmes/emt/key_documents/ emt_competences_translators_fr.pdf> [consulté le $1^{\mathrm{er}}$ juin 2011].

9. En France, grâce notamment aux travaux publiés par le GERAS, Groupe d'étude et de recherche en anglais de spécialité, et à la revue ASp (<http://asp.revues.org>). 
10. On notera, par exemple, les nombreuses rencontres organisées par l'université de Haute Bretagne (Rennes 2) pionnière en la matière (Journées d'études sur la traduction professionnelle, les dernières en juin 2007 et 2009) ; ainsi que celles organisées par l'université de Strasbourg (colloque «Traduction des normes et normes de traduction dans l'espace européen : institutions et entreprises », juin 2010), de Lille (« Traduction, évaluation, qualité », février 2010 ; juin 2011), de Paris-Diderot («Traductologie de plein champ», septembre 2009) et de Grenoble («Traduction et Ergonomie », octobre 2010).

11. Candidature OPTIMALE pour l'appel à proposition DG EAC/41/09, partie C.1.3. Pour en savoir plus sur OPTIMALE : <http://www.translator-training.eu/optimale/> [consulté le $1^{\text {er }}$ juin 2011].

12. Le projet TRACE (TRaducción Asistida, Calidad y Evaluación/Assisted Translation, quality and evaluation, MEC, HUM/2006-04346/FIL) cherche à évaluer, entre autres, les différences entre traductions faites à l'aide de TAO et traductions effectuées sans TAO, à partir d'un corpus de traductions élaborées par les même traducteurs : <http://grupsderecerca.uab.cat/tradumatica/ en/content/trace-traducción-asistida-calidad-y-evaluación> [consulté le $1^{\text {er }}$ juin 2011].

\section{RÉSUMÉS}

Afin de mieux former les traducteurs de demain, il est indispensable de connaître et d'anticiper l'évolution des métiers de la traduction. Si de nombreuses formations de traducteurs ont intégré l'approche par compétences tout en valorisant les compétences professionnelles spécifiques, la traductologie accordait jusqu'à présent peu d'importance aux recherches axées sur les métiers. L'ergonomie offre un cadre de référence qui permet d'analyser les aspects matériels, physiques, cognitifs et organisationnels du métier de traducteur et leur évolution. Le présent article en précise les méthodes et les applique à la traduction, en donnant des pistes pour une traductologie fonctionnelle qui soit attentive à tous les aspects ergonomiques de la traduction.

It is necessary to know and anticipate the changes occurring in the translation profession in order to improve translator education and training. Translation programmes have mostly adopted translation competence models and profiles and tend to focus on specific professional competences, but translation studies so far had not paid much attention to translation profession research. Ergonomics gives a reference framework which makes it possible to analyse the material, physical, cognitive and organisational aspects of the translation profession and their evolution. This paper describes ergonomics methods and applies them to translation practices, showing the way towards functional and ergonomically aware translation studies.

\section{INDEX}

Keywords : cognitive ergonomics, ergonomics, translation, translator training

Mots-clés : ergonomie, ergonomie cognitive, formation de traducteurs, traduction 


\section{AUTEUR}

\section{ÉLISABETH LAVAULT-OLLÉON}

Université Stendhal - Grenoble 3, équipe ILCEA (GREMUTS) 